PROCEEDINGS OF THE

AMERICAN MATHEMATICAL SOCIETY

Volume 139, Number 11, November 2011, Pages 4073-4086

S 0002-9939(2011)10835-9

Article electronically published on April 12, 2011

\title{
DUAL REPRESENTATION OF MONOTONE CONVEX FUNCTIONS ON $L^{0}$
}

\author{
MICHAEL KUPPER AND GREGOR SVINDLAND
}

(Communicated by Richard Rochberg)

\begin{abstract}
We study monotone convex functions $\psi: L^{0}(\Omega, \mathcal{F}, \mathbb{P}) \rightarrow(-\infty, \infty]$ and derive a dual representation as well as conditions that ensure the existence of a $\sigma$-additive subgradient. The results are motivated by applications in economic agents' choice theory.
\end{abstract}

\section{INTRODUCTION}

One of the main concerns in economic theory is the question of economic agents' choice in a set of future random payoffs. This choice behavior is determined by the preferences of each economic agent which may largely differ if one agent is compared to another. However, since e.g. equilibrium and pricing theory are based on such a comparison of agents' preferences, the mathematical analysis of these structures requires not only a description of the agents' preferences in terms of functions but also that these functions be defined on a space of random variables large enough to cover all possible desires the different agents may have. Here the random variables over a probability space $(\Omega, \mathcal{F}, \mathbb{P})$ resemble all possible future random payoffs, and hence a natural underlying space for studying agents' choice behavior is $L^{0}:=L^{0}(\Omega, \mathcal{F}, \mathbb{P})$, the space of all random variables over $(\Omega, \mathcal{F}, \mathbb{P})$ modulo $\mathbb{P}$-almost sure equality. Unfortunately, this space provides some mathematical difficulties as it is not locally convex. Thus, for tractability reasons, the model space is usually restricted to subspaces of $L^{0}$ such as $L^{p}(\Omega, \mathcal{F}, \mathbb{P}), p \in[1, \infty)$, or even further to the space of bounded random variables $L^{\infty}(\Omega, \mathcal{F}, \mathbb{P})$. But the $L^{p}$-spaces, $p \in[1, \infty)$, highly depend on the reference probability measure $\mathbb{P}$. However, when different agents in the market base their respective evaluations on applying different references probability measures respectively or when optimizing the preferences of some agent under constraints given by some pricing rules, it is in general not clear which reference probability one should choose and on which (locally convex) model space the analysis should be carried out. A very common choice of model space is $L^{\infty}$, since it, like $L^{0}$, is invariant under equivalent measure transformations, so the reference probability measure problem does not appear (as long as all candidates

Received by the editors April 22, 2010 and, in revised form, October 7, 2010.

2010 Mathematics Subject Classification. Primary 46A16, 46A20, 49N15, 91 G99.

Key words and phrases. Monotone convex function, duality, subgradient, bipolar representation.

The first author gratefully acknowledges financial support from the MATHEON project E.11.

The second author gratefully acknowledges support from Swissquote.

(C)2011 American Mathematical Society Reverts to public domain 28 years from publication 4073 
for such references probabilities are equivalent). But obviously $L^{\infty}$ is a very limited space, keeping in mind that standard models for prices involve e.g. log-normal distributed random variables (under some pricing probability measure), which thus cannot be incorporated in a setting building on $L^{\infty}$.

Fortunately, it is observed that many common preference functions possess convexity and monotonicity properties which indeed allow for a mathematical inspection of these preferences on the space $L^{0}$. Since this feature is very desirable for the reasons already mentioned, we, during this paper, determine a class of monotone convex functions on $L^{0}$ which exhibit properties adequate for allowing for a dual representation on $L^{0}$ and, under some further conditions, even for a $\sigma$-additive subgradient. This extends, amongst others, results by Brannath and Schachermayer [2] which were formulated on the basis of the same motives as ours. For further ongoing research on topological and structural properties of $L^{0}$ we refer to [9, 11, 12, 14, 15].

The structure of this paper is as follows. Our main results are collected in section 2. In section 2.1] we present a dual representation result for monotone convex functions on $L^{0}$ satisfying in addition a closedness and monotone continuity condition. Based on this result, we derive a bipolar representation for a class of monotone convex subsets of $L^{0}$ which is a generalization of the bipolar theorem presented in 2]. Moreover, in section 2.2 we show that under some additional conditions on the function we obtain a $\sigma$-additive subgradient for a large class of points. These results are then illustrated by examples in section 2.3 The proof of the subgradient result, which is given in section 4, is based on the construction of an auxiliary Banach space which is induced by the monotone convex function studied. This space is defined and studied in section 3 .

\section{DuAl REPRESEntation AND $\sigma$-ADDitive SUbGRADIENTS}

Throughout this paper, let $(\Omega, \mathcal{F}, \mathbb{P})$ be a fixed probability space. For $p \in[0, \infty]$, we write $L^{p}:=L^{p}(\Omega, \mathcal{F}, \mathbb{P})$ and $L^{p}(\mathbb{Q}):=L^{p}(\Omega, \mathcal{F}, \mathbb{Q})$, where $\mathbb{Q}$ is a probability measure absolutely continuous with respect to $\mathbb{P}(\mathbb{Q} \ll \mathbb{P})$. As usual, for two probability measures $\mathbb{Q}$ and $\widehat{\mathbb{Q}}$ on $(\Omega, \mathcal{F})$ we write $\mathbb{Q} \sim \widehat{\mathbb{Q}}$ if and only if $\mathbb{Q} \ll \widehat{\mathbb{Q}}$ and $\widehat{\mathbb{Q}} \ll \mathbb{Q}$. The expectation with respect to $\mathbb{P}$ is denoted by $E[\cdot]$, whereas the expectation with respect to $\mathbb{Q} \ll \mathbb{P}$ is denoted by $E_{\mathbb{Q}}[\cdot]$. We use the convention that $-\infty+\infty:=+\infty-\infty:=-\infty$ and $E[Y]:=E\left[Y^{+}\right]-E\left[Y^{-}\right]$for any $Y \in L^{0}$. All equalities and inequalities between random variables are understood in the $\mathbb{P}_{-}$ almost sure (a.s.) sense. We write $X^{+}:=X \vee 0, X^{-}:=-(X \wedge 0)$ and $L_{+}^{0}:=\left\{X^{+} \mid\right.$ $\left.X \in L^{0}\right\}$, and similarly for the positive (and negative) cones of other spaces. The topology on $L^{0}$ is the convergence in probability which corresponds to the metric $d(X, Y):=E[|X-Y| \wedge 1]$. Recall that this topology is not locally convex and there do not exist non-trivial continuous linear functions from $L^{0}$ to $\mathbb{R}$. So in particular, the classical separation results cannot be applied.

\subsection{A representation result.}

Theorem 2.1. Let $\psi: L^{0} \rightarrow(-\infty,+\infty]$ be a function which exhibits the following properties:

convexity: $\psi(\lambda X+(1-\lambda) Y) \leq \lambda \psi(X)+(1-\lambda) \psi(Y)$ for all $\lambda \in(0,1)$, monotonicity: $X \geq Y$ implies $\psi(X) \geq \psi(Y)$, 
lower semi-continuity (l.s.c.): The level sets $E_{k}:=\left\{X \in L^{0} \mid \psi(X) \leq k\right\}$ are closed (w.r.t. convergence in probability) for all $k \in \mathbb{R}$,

monotone continuity: $\lim _{n \rightarrow \infty} \psi(X \vee(-n))=\psi(X)$ for all $X \in \operatorname{dom} \psi$ which are bounded from above where $\operatorname{dom} \psi:=\left\{Y \in L^{0} \mid \psi(Y)<\infty\right\}$.

Then, $\psi$ has the dual representation

$$
\psi(X)=\sup _{Z \in L_{+}^{\infty}}\left(E[Z X]-\psi^{*}(Z)\right), \quad X \in L^{0},
$$

for the convex conjugate

$$
\begin{aligned}
\psi^{*}(Z) & :=\sup _{X \in L^{0}}(E[Z X]-\psi(X)) \\
& =\sup _{X \in L^{\infty}}(E[Z X]-\psi(X)), \quad Z \in L^{\infty} .
\end{aligned}
$$

The level sets $Q_{k}:=\left\{Z \in L_{+}^{\infty} \mid \psi^{*}(Z) \leq k\right\}, k \in \mathbb{R}$, are convex and closed in the $\sigma\left(L^{\infty}, L^{\infty}\right)$-topology on $L^{\infty}$. If $\operatorname{dom} \psi \neq \emptyset$, then $\psi^{*}>-\infty$.

Remark 2.2. Let $\psi$ be as in Theorem 2.1. Then, the monotonicity and l.s.c. of $\psi$ imply that

$$
\limsup _{m \rightarrow \infty} \psi(X \wedge m) \leq \psi(X) \leq \liminf _{m \rightarrow \infty} \psi(X \wedge m),
$$

so $\psi(X)=\lim _{m \rightarrow \infty} \psi(X \wedge m)$ for all $X \in L^{0}$. Hence, by monotone continuity we infer for every $X \in \operatorname{dom} \psi$ that

$$
\begin{aligned}
\psi(X) & =\lim _{m \rightarrow \infty} \psi(X \wedge m) \\
& =\lim _{m \rightarrow \infty} \lim _{n \rightarrow \infty} \psi((-n) \vee X \wedge m) .
\end{aligned}
$$

We will frequently make use of this continuity property. Moreover, we note that a property similar to (2.3) is used in [5] and [3] in order to extend functions from $L^{\infty}$ to $L^{0}$. More precisely, [5] and [3] give conditions for a class of monotone $\sigma\left(L^{\infty}, L^{1}\right)$-l.s.c. functions $\widehat{\psi}: L^{\infty} \rightarrow \mathbb{R}$ such that the extension given by

$$
\widehat{\psi}(X):=\lim _{n \rightarrow \infty} \lim _{m \rightarrow \infty} \widehat{\psi}((-n) \vee X \wedge m)
$$

maps $L^{0}$ to $(-\infty,+\infty]$. Notice that the limits in (2.3) and (2.4) cannot be interchanged in general. However, in case that $\widehat{\psi}$ is l.s.c. on $L^{0}$ it follows that (2.4) implies (2.3).

Remark 2.3. We like to emphasize that given any function $\psi$ as in Theorem 2.1. in order to obtain the dual representation (2.1) of $\psi$ on $L^{0}$, it suffices to compute the ordinary dual representation of $\psi$ restricted to the Banach space $\left(L^{\infty},\|\cdot\|_{\infty}\right)$ where $\|\cdot\|_{\infty}:=\operatorname{ess} \sup |\cdot|$, because $\psi^{*}$ in (2.2) is the convex conjugate of $\left.\psi\right|_{L^{\infty}}$ restricted to $L^{\infty}$.

Remark 2.4. If we drop the monotone continuity, we may still obtain a representation of type (2.1) for an appropriate $\psi^{*}$. However, the crucial l.s.c. property of $\psi^{*}$, which is essential when translating a primal optimization problem into a dual one, might be lost. For illustration, consider for instance the function

$$
\psi: L^{0} \ni Y \mapsto \delta(Y \mid C):= \begin{cases}0 & \text { if } Y \in C, \\ +\infty & \text { else, }\end{cases}
$$

where $C=\{\hat{X}\}-L_{+}^{0}$ for a point $\hat{X} \in L_{-}^{0}$ such that $E[\hat{X}]=-\infty$. Then, $\psi$ satisfies all conditions required in Theorem 2.1 except for the monotone continuity. It is 
easily verified that $\psi^{*}(Z)=E[Z \hat{X}]$ for all $Z \in L_{+}^{\infty}$. Hence, $\psi^{*}(Z)=-\infty$ for every $Z \in L_{+}^{\infty}$ such that $Z \geq \epsilon$ for some $\epsilon>0$, whereas e.g. $\psi^{*}\left(Z_{\hat{X}}\right)>-\infty$ for $Z_{\hat{X}}:=\frac{1}{1+|\hat{X}|} \in L_{+}^{\infty}$. Therefore, $\psi^{*}$ does not satisfy (2.2) because $\left.\psi\right|_{L^{\infty}}=\infty$, and thus $\sup \left\{E[Z Y]-\psi(Y) \mid Y \in L^{\infty}\right\}=-\infty$ for all $Z \in L_{+}^{\infty}$. In particular the level sets of $\psi^{*}$ are not closed in any topology on $L^{\infty}$ weaker than the norm topology induced by $\|\cdot\|_{\infty}$, because for every neighborhood of $Z_{\hat{X}}$ in the norm topology there is some $n \in \mathbb{N}$ such that $Z_{n}:=Z_{\hat{X}} \vee \frac{1}{n}$ lies in that neighborhood. But, as shown above, $\psi^{*}\left(Z_{n}\right)=-\infty$ for all $n \in \mathbb{N}$, whereas $\psi^{*}\left(Z_{\hat{X}}\right)>-\infty$.

Remark 2.5. Notice that any $\psi: L^{0} \rightarrow[-\infty,+\infty]$ which satisfies the conditions of Theorem 2.1 and for which $\operatorname{dom} \psi \neq \emptyset$ has to be proper (i.e. $\psi>-\infty$ and $\operatorname{dom} \psi \neq \emptyset$ ). Hence, it has a robust representation of the form (2.1). Indeed, if we had $\psi(Y)=-\infty$ for some $Y \in L^{0}$, then we could choose $\mathbb{Q} \sim \mathbb{P}$ such that $Y, X \in L^{1}(\mathbb{Q})$ for some $X \in \operatorname{dom} \psi$. However, since $\left.\psi\right|_{L^{1}(\mathbb{Q})}$ is a convex l.s.c. (w.r.t. $\left.E_{\mathbb{Q}}[|\cdot|]\right)$ function on $L^{1}(\mathbb{Q})$, this would contradict the well-known fact that if such a function assumes the value $-\infty$, it cannot take any finite value (see Proposition 2.4 in $[8])$.

Proof of Theorem 2.1. If $\operatorname{dom} \psi=\emptyset$, i.e. $\psi \equiv \infty$, then $\psi^{*} \equiv-\infty$, so the representation (2.1) holds. In the following we assume that $\operatorname{dom} \psi \neq \emptyset$, which in particular implies that $\psi^{*}>-\infty$, because for every $Z \in L_{+}^{\infty}$ and some $Y \in \operatorname{dom} \psi$ monotonicity implies that $-Y^{-} \in \operatorname{dom} \psi$, and by monotone continuity there must be an $n \in \mathbb{N}$ such that $\psi\left(-\left(Y^{-} \wedge n\right)\right)<\infty$, and thus $E\left[-Z\left(Y^{-} \wedge n\right)\right]-\psi\left(-\left(Y^{-} \wedge n\right)\right)>-\infty$. Next we show that

$$
\forall X \in L^{0}: \quad \psi(X) \geq \sup _{Z \in L_{+}^{\infty}}\left(E[Z X]-\psi^{*}(Z)\right) .
$$

This assertion is trivial if $\psi(X)=\infty$ or if $Z \in L_{+}^{\infty}$ is such that $E[Z X]=-\infty$ or $\psi^{*}(Z)=\infty$ or both. Hence, the only case that remains is $X \in \operatorname{dom} \psi,-\infty<$ $E[Z X]<\infty$, and $\psi^{*}(Z)<\infty$ (note that $X \in \operatorname{dom} \psi$ and $E[Z X]=\infty$ would imply $\psi^{*}(Z)=\infty$ ), and in this case, by definition of $\psi^{*}$, we have

$$
E[Z X]-\psi^{*}(Z) \leq E[Z X]-E[Z X]+\psi(X)=\psi(X)
$$

so (2.5) is verified. Now consider any $X \in L^{0}$. Then $X \in L^{1}(\mathbb{Q})$ for $\mathbb{Q} \sim \mathbb{P}$ given by

$$
\frac{d \mathbb{Q}}{d \mathbb{P}}=\frac{1}{C(1+|X|)} \in L_{+}^{\infty}, \quad \text { where } \quad C=E\left[\frac{1}{1+|X|}\right] .
$$

Note that either $\left.\psi\right|_{L^{1}(\mathbb{Q})} \equiv \infty$ or $\left.\psi\right|_{L^{1}(\mathbb{Q})}$ is a proper convex function which is l.s.c. with respect to $\|\cdot\|_{L^{1}(\mathbb{Q})}=E_{\mathbb{Q}}[|\cdot|]$. In either case, the Fenchel-Moreau theorem (see Proposition 3.1 in [8]) and the monotonicity of $\psi$ yield

$$
\psi(X)=\sup _{Z \in L_{+}^{\infty}}\left(E\left[\frac{d \mathbb{Q}}{d \mathbb{P}} Z X\right]-\left.\psi\right|_{L^{1}(\mathbb{Q})} ^{*}(Z)\right)
$$

where

$$
\left.\psi\right|_{L^{1}(\mathbb{Q})} ^{*}(Z)=\sup _{Y \in L^{1}(\mathbb{Q})}\left(E\left[\frac{d \mathbb{Q}}{d \mathbb{P}} Z Y\right]-\psi(Y)\right), \quad Z \in L^{\infty} .
$$

Next we show that

$$
\left.\psi\right|_{L^{1}(\mathbb{Q})} ^{*}(Z)=\psi^{*}\left(\frac{d \mathbb{Q}}{d \mathbb{P}} Z\right)=\sup _{Y \in L^{\infty}}\left(E\left[\frac{d \mathbb{Q}}{d \mathbb{P}} Z Y\right]-\psi(Y)\right) \quad \text { for all } Z \in L^{\infty}
$$


Indeed, by definition it is obvious that for all $Z \in L^{\infty}$,

$$
\sup _{Y \in L^{\infty}}\left(E\left[\frac{d \mathbb{Q}}{d \mathbb{P}} Z Y\right]-\psi(Y)\right) \leq\left.\psi\right|_{L^{1}(\mathbb{Q})} ^{*}(Z) \leq \psi^{*}\left(\frac{d \mathbb{Q}}{d \mathbb{P}} Z\right) .
$$

Hence, noting that $\frac{d \mathbb{Q}}{d \mathbb{P}} \in L_{+}^{\infty}$, the assertion will follow if we show that

$$
\psi^{*}(Z) \leq \sup _{Y \in L^{\infty}}(E[Z Y]-\psi(Y)) \quad \text { for all } Z \in L^{\infty} .
$$

To this end, let $Z \in L^{\infty}$ and $Y \in \operatorname{dom} \psi$ such that $E[Z Y]>-\infty$. If $E[Z Y]<\infty$, then for every $\epsilon>0$, by monotone convergence and Remark 2.2 , there exist $n, m \in \mathbb{N}$ such that $Y_{n, m}:=(-n) \vee Y \wedge m \in L^{\infty}$ satisfies $E[Y Z] \leq E\left[Z Y_{n, m}\right]+\epsilon$ and $-\psi(Y) \leq-\psi\left(Y_{n, m}\right)+\epsilon$ so that

$$
E[Z Y]-\psi(Y) \leq E\left[Z Y_{n, m}\right]-\psi\left(Y_{n, m}\right)+2 \epsilon,
$$

and (2.8) follows. If $E[Z Y]=\infty$, then again by monotone convergence and Remark 2.2, for every $N \in \mathbb{N}$ and $\epsilon>0$ there exist $n, m \in \mathbb{N}$ such that $Y_{n, m}:=$ $(-n) \vee Y \wedge m \in L^{\infty}$ satisfies $E\left[Z Y_{n, m}\right] \geq N$ and $-\psi(Y) \leq-\psi\left(Y_{n, m}\right)+\epsilon$, which yields

$$
N-\psi(Y)-\epsilon \leq E\left[Z Y_{n, m}\right]-\psi\left(Y_{n, m}\right),
$$

and (2.8) is verified in this case too. Consequently, (2.2) and (2.7) are proved. In conjunction with (2.5) and (2.6) we obtain

$$
\begin{aligned}
\psi(X) & =\sup _{Z \in L_{+}^{\infty}}\left(E\left[\frac{d \mathbb{Q}}{d \mathbb{P}} Z X\right]-\left.\psi\right|_{L^{1}(\mathbb{Q})} ^{*}(Z)\right)=\sup _{Z \in L_{+}^{\infty}}\left(E\left[\frac{d \mathbb{Q}}{d \mathbb{P}} Z X\right]-\psi^{*}\left(\frac{d \mathbb{Q}}{d \mathbb{P}} Z\right)\right) \\
& \leq \sup _{Z \in L_{+}^{\infty}}\left(E[Z X]-\psi^{*}(Z)\right) \leq \psi(X),
\end{aligned}
$$

and (2.1) is proved. Finally notice that according to (2.2), the convex function $\psi^{*}$ is $\sigma\left(L^{\infty}, L^{\infty}\right)$-l.s.c., which means that the level sets $Q_{k}$ are convex and closed in the $\sigma\left(L^{\infty}, L^{\infty}\right)$-topology. Thus the theorem is proved.

From Theorem 2.1 we derive the following bipolar theorem.

Theorem 2.6. Let $C$ be a closed convex subset of $L^{0}$ such that $0 \in C$ and $C-L_{+}^{0} \subset$ $C$. Moreover, suppose that for any $X \in C$ there is $n \in \mathbb{N}$ such that $X \vee(-n) \in C$. Then,

$$
X \in C \quad \Longleftrightarrow \quad \forall Z \in C^{\circ}: E[Z X] \leq 1,
$$

where the polar $C^{\circ}:=\left\{Z \in L_{+}^{\infty} \mid \forall X \in C: E[Z X] \leq 1\right\}$ of $C$ is a convex set with $0 \in C^{\circ}$ which is closed in the $\sigma\left(L^{\infty}, L^{\infty}\right)$-topology on $L^{\infty}$.

Proof. The indicator function $\delta(\cdot \mid C)$ satisfies the conditions of Theorem 2.1] Hence, we obtain

$$
X \in C \quad \Longleftrightarrow \quad \forall Z \in L_{+}^{\infty}: E[Z X] \leq \delta^{*}(Z \mid C)
$$

for the convex conjugate

$$
\delta^{*}(Z \mid C):=\delta(\cdot \mid C)^{*}(Z)=\sup _{X \in L^{0}}(E[Z X]-\delta(X \mid C))=\sup _{X \in C} E[Z X],
$$

which coincides with the support function of $C$. Since $C^{\circ}$ equals the level set $Q_{1}=\left\{Z \in L_{+}^{\infty} \mid \delta^{*}(Z \mid C) \leq 1\right\}$ of $\delta^{*}(\cdot \mid C)$, we infer from Theorem 2.1 that $C^{\circ}$ is $\sigma\left(L^{\infty}, L^{\infty}\right)$-closed and convex, whereas $0 \in C^{\circ}$ is obvious. The fact that $X \in C$ implies that the right hand side in (2.9) follows directly from the definition of the 
polar $C^{\circ}$. In the case that $X \notin C$ it follows from (2.10) that there is $\bar{Z} \in L_{+}^{\infty}$ such that

$$
\infty \geq E[\bar{Z} X]>\delta^{*}(\bar{Z} \mid C) \geq 0,
$$

where the latter inequality is due to $0 \in C$. We distinguish two different cases. On the one hand, if $\delta^{*}(\bar{Z} \mid C)>0$, it follows that $E[\hat{Z} X]>1$ for $\hat{Z}:=\bar{Z} / \delta^{*}(\bar{Z} \mid$ $C) \in C^{\circ}$. On the other hand, if $\delta^{*}(\bar{Z} \mid C)=0$, it follows that $\delta^{*}(n \bar{Z} \mid C)=0$ and therefore $n \bar{Z} \in C^{\circ}$ for all $n \in \mathbb{N}$. In particular, for $n$ large enough we deduce that $E[(n \bar{Z}) X]>1$, and the proof is completed.

Restricting the results of Theorem 2.6 to $L_{+}^{0}$ yields the bipolar theorem of Brannath and Schachermayer [2].

Corollary 2.7. Let $C$ denote a closed convex subset of $L_{+}^{0}$ which is solid; i.e. $X \in C$ and $0 \leq Y \leq X$ implies $Y \in C$. Then

$$
X \in C \quad \Longleftrightarrow \quad X \geq 0 \text { and } \forall Z \in C^{\circ}: E[Z X] \leq 1
$$

for the polar $C^{\circ}=\left\{Z \in L_{+}^{\infty} \mid \forall X \in C: E[Z X] \leq 1\right\}$ which is a convex $\sigma\left(L^{\infty}, L^{\infty}\right)$ closed subset of $L^{\infty}$ such that $0 \in C^{\circ}$.

Proof. Define $\tilde{C}:=C-L_{+}^{0}$ which is convex. Note that for any $X \in \tilde{C}$ we have $X^{+} \in C \subset \tilde{C}$ by solidity. Moreover, $\tilde{C}$ is closed since for any sequence $\left(X_{n}\right)_{n \in \mathbb{N}} \subset \tilde{C}$ which converges in probability to $X \in L^{0}$, it follows that $\left(X_{n}^{+}\right)_{n \in \mathbb{N}} \subset C$ converges in probability to $X^{+}$and thus $X^{+} \in C$ by closedness of $C$. Hence, $X \in \tilde{C}$. Since

$$
\tilde{C}^{\circ}=\left\{Z \in L_{+}^{\infty} \mid \forall X \in \tilde{C}: E[Z X] \leq 1\right\}=\left\{Z \in L_{+}^{\infty} \mid \forall X \in C: E[Z X] \leq 1\right\},
$$

the claim follows from Theorem 2.6.

Remark 2.8. In 2] Brannath and Schachermayer proved the assertion of Corollary 2.7 for a different polar, namely,

$$
\widetilde{C}^{\circ}=\left\{Z \in L_{+}^{0} \mid \forall X \in C: E[Z X] \leq 1\right\} \subset L^{0}
$$

instead of $C^{\circ} \subset L_{+}^{\infty}$, as given in Corollary 2.7. Note that our result is stronger since $C^{\circ} \subset \widetilde{C}^{\circ}$, and thus the non-trivial implication

$$
X \geq 0 \text { and } \forall Z \in \widetilde{C}^{\circ}: E[Z X] \leq 1 \quad \Rightarrow \quad X \in C
$$

immediately follows once we know (2.11).

2.2. $\sigma$-additive subgradients. In order to obtain a maximizer in the dual representation (2.1) we will have to enlarge the space of test measures from $L_{+}^{\infty}$ to $L_{+}^{1}$ and impose further conditions on $\psi$. Theorem 2.9 states conditions under which there is such a maximizer, which can be seen as a kind of generalized subgradient. The proof of Theorem 2.9 is given in section 4 .

Theorem 2.9. Let $\psi: L^{0} \rightarrow(-\infty, \infty]$ be as in Theorem 2.1 , and suppose that $\psi$ in addition satisfies:

normalization: $\psi(0)=0$ and $\mathbb{R} \subset \operatorname{dom} \psi$, sensitivity: $\psi(|X|)>0$ for all $X \in L^{0} \backslash\{0\}$, and

bounded continuity: if $\left(X_{n}\right)_{n \in \mathbb{N}} \subset L^{\infty}$ and $X \in L^{\infty}$ such that $X_{n} \downarrow X \mathbb{P}$-a.s., then $\psi\left(X_{n}\right) \downarrow \psi(X)$. 
Then for every $X \in L^{0}$ such that there is an $\epsilon>0$ with

$$
\psi\left((1+\epsilon) X^{+}\right)<\infty \text { and } \lim _{n \rightarrow \infty} \psi\left(X+\epsilon X 1_{\{X \geq n\}}\right)=\psi(X),
$$

we have

$$
\psi(X)=\max _{Z \in L_{+}^{1}}\left(E[Z X]-\psi^{*}(Z)\right)
$$

where

$$
\psi^{*}(Z)=\sup _{Y \in L^{\infty}}(E[Z Y]-\psi(Y)), \quad Z \in L^{1} .
$$

Note that the convex conjugate $\psi^{*}$ appearing in Theorem 2.9 is the extension to $L^{1}$ of the convex conjugate given in Theorem 2.1 hence the same denotation.

Remark 2.10. The fact that we need the property of bounded continuity in Theorem 2.9 is illustrated by considering $\psi(X):=\operatorname{ess} \sup X, X \in L^{0}$. This function satisfies all conditions required in Theorem 2.1, and the dual representation is

$$
\psi(X)=\sup _{Z \in L_{+}^{\infty}}\left(E[Z X]-\psi^{*}(Z)\right)=\sup _{Z \in L_{+}^{1}}\left(E[Z X]-\psi^{*}(Z)\right),
$$

where $\psi^{*}=\delta(\cdot \mid \mathcal{Q})$ for $\mathcal{Q}=\left\{Z \in L_{+}^{1} \mid E[Z]=1\right\}$. Moreover, $\psi$ is normalized and sensitive, but fails to be bounded continuous. Indeed, for an $X \in L^{0}$ such that ess sup $X<\infty$, but $\mathbb{P}(X=\operatorname{ess} \sup X)=0$, there cannot exist an $Z \in \mathcal{Q}$ such that $E[Z X]=\operatorname{ess} \sup X$.

2.3. Examples. In this subsection we illustrate the main theorems, Theorems 2.1 and 2.9.

Example 2.11. (Entropic risk measure) Define $\psi(X):=\log E[\exp (X)], X \in L^{0}$, so that $\psi(-X)$ is the entropic risk measure and $-\psi(-X)$ corresponds to the certainty equivalent of an exponential expected utility function. $\psi$ is a convex and monotone function which maps $L^{0}$ to $(-\infty,+\infty]$. Moreover, $\psi$ is l.s.c. and monotone continuous due to Fatou's lemma and the dominated convergence theorem, respectively. According to theorem 2.1 the function $\psi$ has the dual representation (2.1) for a convex conjugate $\psi^{*}$, the domain of which is concentrated on the probability densities and which is given by $\psi^{*}(Z)=E[Z \log (Z)]$ for $Z \in L_{+}^{\infty}$ with $E[Z]=1$. For an explicit computation of $\psi^{*}$ we refer to [10, keeping Remark 2.3 in mind. The entropic risk measure belongs to the class of optimized certainty equivalents, dual representations of which have been studied on $L^{0}$ in [4].

As for the subgradients, we notice that the function $\psi$ is normalized, sensitive and bounded continuous. Also, for $X \in L^{0}$ with $(1+\epsilon) X \in \operatorname{dom} \psi$ or equivalently $(1+\epsilon) X^{+} \in \operatorname{dom} \psi$ for some $\epsilon>0$, it follows that $\psi\left(X+\epsilon X 1_{\{X \geq n\}}\right)$ converges to $\psi(X)$ for $n \rightarrow \infty$ due to the dominated convergence Theorem. Thus, we derive from Theorem 2.9 the existence of a subgradient for $\psi$ at $X$, i.e. the maximizer of (2.12), which directly computes as $\exp (X) / E[\exp (X)] \in L^{1}$.

Example 2.12. (Economic index of riskiness) Let $l: \mathbb{R} \rightarrow[c,+\infty)$ be an l.s.c. strictly increasing convex loss function such that $l(0)>0$, and one which is bounded from below. We choose an acceptability threshold $c_{0}>l(0)$ and define

$$
\psi(X):=\inf \left\{t>0 \mid E\left[l\left(\frac{X}{t}\right)\right] \leq c_{0}\right\}, \quad X \in L^{0} \quad(\inf \emptyset:=\infty) .
$$


Following [6], the economic index of riskiness is defined as $\rho(X):=\psi(-X)$. Note that $\psi(X)=0$ if $X \leq 0$, since by monotonicity of $l$ we have $E[l(X / t)] \leq l(0)<c_{0}$ for all $t>0$. As $\psi$ is a gauge function, it is sublinear, so in particular convex. Moreover, the dominated convergence theorem and Fatou's lemma show that $\psi$ satisfies the conditions of Theorem 2.1. We claim that the dual representation of $\psi$ is given by

$$
\psi(X)=\sup _{Z \in L_{+}^{\infty}} \frac{E[Z X]}{c_{0}+E\left[l^{*}(Z)\right]}=\sup _{Z \in L_{+}^{\infty}}(E[Z X]-\delta(Z \mid \mathcal{Q})) \quad \text { for all } X \in L^{0},
$$

where $\mathcal{Q}$ denotes the $\sigma\left(L^{\infty}, L^{\infty}\right)$-closure of the convex hull of

$$
\left\{\frac{Z}{c_{0}+E\left[l^{*}(Z)\right]} \mid Z \in L_{+}^{\infty}\right\}
$$

and $l^{*}(z)=\sup \{z x-l(x) \mid x \in \mathbb{R}\}, z \in \mathbb{R}$, is the convex conjugate of $l$. Indeed, according to Remark 2.3, in order to obtain the dual representation of $\psi$ we only need to derive the dual representation of $\left.\psi\right|_{L^{\infty}}$, and for any $X \in L^{\infty}$ it follows that

$$
\begin{aligned}
\psi(X) & =\inf \left\{t>0 \mid E\left[\sup _{z \in \mathbb{R}}\left\{z \frac{X}{t}-l^{*}(z)\right\}\right] \leq c_{0}\right\} \\
& =\inf \left\{t>0 \mid \forall Z \in L_{+}^{\infty}: E[Z X] \leq t\left(c_{0}+E\left[l^{*}(Z)\right]\right)\right\} .
\end{aligned}
$$

As for the subgradients, we notice that the function $\psi$ is normalized, sensitive and bounded continuous. Let $X \in L^{0}$ with $(1+\epsilon) X^{+} \in \operatorname{dom} \psi$ for some $\epsilon>0$ and suppose that $E\left[l\left((1+\epsilon) X^{+} / \psi(X)\right)\right]<\infty$. Then the dominated convergence theorem yields $\lim _{n \rightarrow \infty} \psi\left(X+\epsilon X 1_{\{X \geq n\}}\right)=\psi(X)$, and Theorem 2.9 implies the existence of a subgradient at $X$. In case $l$ is differentiable, the subgradient of $\psi$ at $X$ is given by

$$
\frac{Z_{X}}{c_{0}+E\left[l^{*}\left(Z_{X}\right)\right]} \in L_{+}^{1} \quad \text { for } \quad Z_{X}=l^{\prime}\left(\frac{X}{\psi(X)}\right)
$$

\section{THE SPACE $L^{\psi}$ AND ITS DUAL $L^{\psi *}$}

In this section we prepare the proof of Theorem 2.9 by introducing and studying a Banach space $L^{\psi}$ which is induced by the function $\psi$ as given in Theorem 2.9. Its dual space $L^{\psi *}$ will provide the desired subgradients. This further develops ideas presented in [17.

Definition 3.1. Let $\psi: L^{0} \rightarrow(-\infty, \infty]$ be a convex function such that $\psi(0)=0$. Then, we define

$$
\|X\|_{\psi}:=\inf \{\lambda>0 \mid \psi(|X| / \lambda) \leq 1\}, \quad X \in L^{0},
$$

with the usual convention that $\inf \emptyset=\infty$ and

$$
L^{\psi}:=\left\{X \in L^{0} \mid\|X\|_{\psi}<\infty\right\} .
$$

Apparently, the idea behind the construction of the $\left(L^{\psi},\|\cdot\|_{\psi}\right)$ space is a generalization of the construction of Orlicz-spaces via Luxemburg-norms.

Proposition 3.2. Let $\psi: L^{0} \rightarrow(-\infty, \infty]$ be a convex, monotone, normalized and sensitive function. Then:

(i) $\|\cdot\|_{\psi}: L^{0} \rightarrow[0, \infty]$ is a sub-linear function. 
(ii) There exists $K>0$ such that $\|X\|_{\psi} \leq K\|X\|_{\infty}$ for all $X \in L^{\infty}$.

(iii) $\left(L^{\psi},\|\cdot\|_{\psi}\right)$ is a normed space such that $L^{\infty} \subset L^{\psi}$ and $L_{\infty}^{\psi *} \subset L^{\infty *}$, where $L^{\psi *}$ and $L^{\infty *}$ denote the dual spaces of $L^{\psi}$ and $L^{\infty}$ respectively, and $L_{\infty}^{\psi *}:=\left\{\left.l\right|_{L^{\infty}} \mid l \in L^{\psi *}\right\}$.

(iv) If $X \in L^{\psi}$, then $|X| \in L^{\psi}$ and $X 1_{A} \in L^{\psi}$ for all $A \in \mathcal{F}$. In particular, $X \in L^{\psi}$ if and only if $X^{+}, X^{-} \in L^{\psi}$. Moreover, if $Y \in L^{0}$ and $X \in L^{\psi}$ such that $|Y| \leq|X|$, then $Y \in L^{\psi}$ and $\|Y\|_{\psi} \leq\|X\|_{\psi}$.

(v) If in addition $\psi$ satisfies

$$
\mathbb{R} \ni \alpha \mapsto \psi(\alpha X) \text { is an l.s.c. function on } \mathbb{R} \text { for every } X \in L^{0},
$$

then $\left.\psi\right|_{L^{\psi}}$ is a proper l.s.c. convex function on $\left(L^{\psi},\|\cdot\|_{\psi}\right)$.

(vi) If $\psi$ is l.s.c. on $L^{0}$, then $\left(L^{\psi},\|\cdot\|_{\psi}\right)$ is a Banach space, and there exists a probability measure $\mathbb{Q}$ on $(\Omega, \mathcal{F})$ with $\mathbb{Q} \sim \mathbb{P}$ such that $L^{\psi} \subset L^{1}(\mathbb{Q})$.

Note that l.s.c. on $L^{0}$ implies (3.1) but not vice versa (see Example 3.3).

Proof. We define $\Lambda(X):=\{\lambda>0 \mid \psi(|X| / \lambda) \leq 1\}, X \in L^{0}$.

(i) It is easily verified that $\|t X\|_{\psi}=|t| \cdot\|X\|_{\psi}$ for all $t \in \mathbb{R}$. In order to show that $\|X+Y\|_{\psi} \leq\|X\|_{\psi}+\|Y\|_{\psi}$ it suffices to consider $X, Y \in L^{\psi}$, because if either $\|X\|_{\psi}=\infty$ or $\|Y\|_{\psi}=\infty$ or both, the assertion is trivial. To this end let $\alpha \in \Lambda(X)$ and $\beta \in \Lambda(Y)$ for some $X, Y \in L^{\psi}$. Then, by monotonicity and convexity

$$
\begin{aligned}
\psi\left(\frac{|X+Y|}{\alpha+\beta}\right) & \leq \psi\left(\frac{\alpha}{\alpha+\beta} \frac{|X|}{\alpha}+\frac{\beta}{\alpha+\beta} \frac{|Y|}{\beta}\right) \\
& \leq \frac{\alpha}{\alpha+\beta} \psi\left(\frac{|X|}{\alpha}\right)+\frac{\beta}{\alpha+\beta} \psi\left(\frac{|Y|}{\beta}\right) \leq 1
\end{aligned}
$$

so $\Lambda(X)+\Lambda(Y) \subset \Lambda(X+Y)$, which proves the triangle inequality.

(ii) and (iii) Clearly, $\|0\|_{\psi}=0$. For all $X \in L^{0} \backslash\{0\}$ and $0<\lambda<1$

$$
\psi\left(\frac{|X|}{\lambda}\right) \geq \frac{1}{\lambda} \psi(|X|) \rightarrow \infty \text { for } \lambda \rightarrow 0,
$$

because $\psi(|X|)>0$ due to sensitivity of $\psi$. Hence, there exists a $\widetilde{\lambda}>0$ such that $\psi(|X| / \lambda)>1$ for all $\lambda \leq \widetilde{\lambda}$, so $\|X\|_{\psi} \geq \widetilde{\lambda}>0$. Consequently, $\|X\|_{\psi}=0$ if and only if $X=0$. Apparently, the properties of $\|\cdot\|_{\psi}$ ensure that $\left(L^{\psi},\|\cdot\|_{\psi}\right)$ is a normed space.

For every $0 \neq X \in L^{\infty}$ and $K \geq 1$ we obtain

$$
\psi\left(\frac{|X|}{K\|X\|_{\infty}}\right) \leq \psi\left(\frac{1}{K}\right) \leq \frac{1}{K} \psi(1)
$$

due to monotonicity and convexity. By normalization there is a $K \geq 1$ such that $\psi\left(\frac{|X|}{K\|X\|_{\infty}}\right) \leq 1$ for all $X \in L^{\infty} \backslash\{0\}$, which means that $\|X\|_{\psi} \leq K\|X\|_{\infty}$, and thus $L^{\infty} \subset L^{\psi}$ and $L_{\infty}^{\psi *} \subset L^{\infty *}$.

(iv) This is obvious by monotonicity of $\psi$.

(v) Obviously, $\psi=\left.\psi\right|_{L^{\psi}}$ is a proper convex function. We only have to establish the l.s.c. of $\psi$ on $\left(L^{\psi},\|\cdot\|_{\psi}\right)$. To this end, let $\left(X_{n}\right)_{n \in \mathbb{N}} \subset L^{\psi}$ and $X \in L^{\psi}$ be such that $\left\|X_{n}-X\right\|_{\psi} \rightarrow 0$. In other words, for every $\lambda>0$ there is $n(\lambda) \in \mathbb{N}$ such that 
for all $n \geq n(\lambda)$ we have $\psi\left(\left|X_{n}-X\right| / \lambda\right) \leq 1$. Then for every $\alpha \in(0,1)$ and all $n \geq n\left(\frac{1-\alpha}{\alpha}\right)$ monotonicity and convexity yield

$$
\begin{aligned}
\psi(\alpha X) & \leq \psi\left((1-\alpha) \frac{\alpha\left|X-X_{n}\right|}{(1-\alpha)}+\alpha X_{n}\right) \\
& \leq(1-\alpha) \psi\left(\frac{\alpha\left|X-X_{n}\right|}{(1-\alpha)}\right)+\alpha \psi\left(X_{n}\right) \leq(1-\alpha)+\alpha \psi\left(X_{n}\right) .
\end{aligned}
$$

Hence, we conclude that for all $\alpha \in(0,1)$ we have

$$
\psi(\alpha X) \leq(1-\alpha)+\alpha \liminf _{n \rightarrow \infty} \psi\left(X_{n}\right) .
$$

By assumption $\mathbb{R} \ni \alpha \mapsto \psi(\alpha X)$ is an l.s.c. function on $\mathbb{R}$. Hence,

$$
\psi(X) \leq \liminf _{\alpha \uparrow 1} \psi(\alpha X) \leq \liminf _{\alpha \uparrow 1}\left((1-\alpha)+\alpha \liminf _{n \rightarrow \infty} \psi\left(X_{n}\right)\right)=\liminf _{n \rightarrow \infty} \psi\left(X_{n}\right) .
$$

(vi) If $\psi$ is l.s.c. on $L^{0}$, so is $\|\cdot\|_{\psi}$, because for every sequence $\left(X_{n}\right)_{n \in \mathbb{N}} \subset L^{0}$ converging in probability to $X \in L^{0}$ and for every $\lambda>0$, the sequence $\left|X_{n}\right| / \lambda$ converges to $|X| / \lambda$ in probability, so $\psi(|X| / \lambda) \leq \liminf _{n \rightarrow \infty} \psi\left(\left|X_{n}\right| / \lambda\right)$, and therefore the level sets $E_{k}^{0}:=\left\{X \in L^{0} \mid\|X\|_{\psi} \leq k\right\}, k \in \mathbb{R}$, are closed. Consider the level sets $E_{k}^{\infty}:=\left\{X \in L^{\infty} \mid\|X\|_{\psi} \leq k\right\}$ of $\|\cdot\|_{\psi}$ viewed as a function on $L^{\infty}$. Since $E_{k}^{0}$ is closed in $L^{0}$, we have that the sets $E_{k}^{\infty} \cap\left\{X \in L^{\infty} \mid\|X\|_{\infty} \leq r\right\}$ are closed in $L^{1}$ for every $r>0$, and thus $E_{k}^{\infty}$ is $\sigma\left(L^{\infty}, L^{1}\right)$-closed in $L^{\infty}$ due to the Krein-Šmulian theorem (see [16, Theorem 5). Consequently, $\|\cdot\|_{\psi}$ viewed as a function on $L^{\infty}$ is $\sigma\left(L^{\infty}, L^{1}\right)$-l.s.c. In particular, by the Fenchel-Moreau theorem (Proposition 3.1 in [8]), we know that

$$
\|X\|_{\psi}=\sup _{Z \in \mathcal{Q}} E[Z X], \quad X \in L^{\infty},
$$

for a convex set $\mathcal{Q} \subset L^{1}$. We may assume that $Z \in \mathcal{Q}$ implies $Z^{+} \in \mathcal{Q}$, because for all $X \in L_{+}^{\infty}$ we have

$$
E\left[Z^{+} X\right] \leq E\left[Z\left(X 1_{\{Z \geq 0\}}-X 1_{\{Z<0\}}\right)\right] \leq\left\|X 1_{\{Z \geq 0\}}-X 1_{\{Z<0\}}\right\|_{\psi}=\|X\|_{\psi} .
$$

Since $\left\|1_{A}\right\|_{\psi}>0$ for every $A \in \mathcal{F}$ such that $\mathbb{P}(A)>0$, we infer that there must be some $Z \in \mathcal{Q} \cap L_{+}^{1}$ such that $E\left[Z 1_{A}\right]>0$. Therefore, if $\mathcal{Q}_{m}$ denotes the set of probabilities $\mathbb{Q}_{Z}$ given by the densities

$$
\frac{d \mathbb{Q}_{Z}}{d \mathbb{P}}=\frac{Z}{E[Z]}, \quad Z \in \mathcal{Q} \cap L_{+}^{1} \backslash\{0\},
$$

then we have that $\mathcal{Q}_{m} \sim \mathbb{P}$ in the sense that

$$
\forall A \in \mathcal{F}:\left[\left(\forall \mathbb{Q} \in \mathcal{Q}_{m}: \mathbb{Q}(A)=0\right) \quad \Leftrightarrow \quad \mathbb{P}(A)=0\right] .
$$

Hence, the Halmos-Savage theorem (see Lemma 7 in [13]) implies that there is a countable subset $\widetilde{\mathcal{Q}}_{m}=\left\{\mathbb{Q}_{z_{1}}, \mathbb{Q}_{z_{2}}, \ldots\right\}$ of $\mathcal{Q}_{m}$ which satisfies $\widetilde{\mathcal{Q}}_{m} \sim \mathbb{P}$. Let

$$
Z:=\sum_{n=1}^{\infty} \frac{1}{2^{n}\left(E\left[Z_{n}\right] \vee 1\right)} Z_{n}
$$

Then, $Z \in L^{1}$ and $Z>0$. Moreover, for every $X \in L^{\infty}$ we have

$$
E[Z X]=\sum_{n=1}^{\infty} \frac{1}{2^{n}\left(E\left[Z_{n}\right] \vee 1\right)} E\left[Z_{n} X\right] \leq \sum_{n=1}^{\infty} \frac{1}{2^{n}\left(E\left[Z_{n}\right] \vee 1\right)}\|X\|_{\psi} \leq\|X\|_{\psi} .
$$


Even more, for every $X \in L^{\psi}$ we obtain

$$
E[Z|X|]=\lim _{n \rightarrow \infty} E[Z(|X| \wedge n)] \leq \limsup _{n \rightarrow \infty}\||X| \wedge n\|_{\psi} \leq\|X\|_{\psi} .
$$

Hence, we infer that $L^{\psi} \subset L^{1}(\mathbb{Q})$, where the probability measure $\mathbb{Q} \sim \mathbb{P}$ is given by $\frac{d \mathbb{Q}}{d \mathbb{P}}=\frac{Z}{E[Z]}$. In particular, we observe that if $\left(X_{n}\right)_{n \in \mathbb{N}}$ is a Cauchy sequence in $\left(L^{\psi},\|\cdot\|_{\psi}\right)$, then it must also be a Cauchy sequence in $\left(L^{1}(\mathbb{Q}), E_{\mathbb{Q}}[|\cdot|]\right)$ which is a complete space. Let $X$ be the limit of $\left(X_{n}\right)_{n \in \mathbb{N}}$ in $\left(L^{1}(\mathbb{Q}), E_{\mathbb{Q}}[|\cdot|]\right)$ and recall that $X_{n}$ thus converges to $X$ in probability, too. Since any Cauchy sequence in a normed space is norm bounded, there exists a $K>0$ such that $\left\|X_{n}\right\|_{\psi} \leq K$ for all $n \in \mathbb{N}$. Therefore, and by l.s.c. of $\|\cdot\|_{\psi}$ on $L^{0}$, we obtain $\|X\|_{\psi} \leq \liminf _{n \rightarrow \infty}\left\|X_{n}\right\|_{\psi} \leq K$, which means that $X \in L^{\psi}$. Moreover, for every $\delta>0$ and $n(\delta) \in \mathbb{N}$ such that $\left\|X_{n}-X_{m}\right\|_{\psi} \leq \delta$, for all $n, m \geq n(\delta)$ we have

$$
\left\|X_{n}-X\right\|_{\psi} \leq \liminf _{m \rightarrow \infty}\left\|X_{n}-X_{m}\right\|_{\psi} \leq \delta \quad \text { if } n \geq n(\delta)
$$

i.e. $X$ is the $\left(L^{\psi},\|\cdot\|_{\psi}\right)$-limit of $\left(X_{n}\right)_{n \in \mathbb{N}}$. Thus we have proved that $\left(L^{\psi},\|\cdot\|_{\psi}\right)$ is complete.

Example 3.3. Consider $\psi(X)=E\left[X^{+}\right]+\delta\left(X^{+} \mid M\right)$, where $M=L^{p}, p \in[0, \infty]$. If $M=L^{p}$ for some $p \in[0,1]$, then $L^{\psi}=L^{1}$ and $\|\cdot\|_{\psi}=E[|\cdot|]$. If $M=L^{p}$ for some $p \in(1, \infty]$, then $L^{\psi}=L^{p}$, but still $\|\cdot\|_{\psi}=E[|\cdot|]$, so $\left(L^{\psi},\|\cdot\|_{\psi}\right)$ is a non-complete normed space. In all cases $\psi$ satisfies (3.1) and thus is a proper l.s.c. convex function on $L^{\psi}$.

Proposition 3.4 is crucial in the proof of Theorem 2.9. as it implies that if $\psi$ satisfies the conditions required in Theorem [2.9, then the intersection $L^{\psi *} \cap L^{1}$ is large enough to allow for $\sigma$-additive subgradients.

Proposition 3.4. Let $\psi: L^{0} \rightarrow(-\infty, \infty]$ be a convex, monotone, normalized and sensitive function which in addition is l.s.c. on $L^{0}$ and satisfies bounded continuity. Then $L_{\infty}^{\psi *} \subset L^{1}$; i.e. any continuous linear functional $\mu$ on $L^{\psi}$ restricted to $L^{\infty}$ is of type $L^{\infty} \ni X \mapsto E\left[Z_{\mu} X\right]$ for a unique $Z_{\mu} \in L^{1}$. Moreover, $Z_{\mu} \in L^{\psi *}$.

Proof. Step 1. First of all, we prove that $\left\|X_{n}-X\right\|_{\psi} \rightarrow 0$ whenever $\left(X_{n}\right)_{n \in \mathbb{N}} \subset L^{\infty}$ is a bounded sequence and $X \in L^{\infty}$ such that $X_{n} \rightarrow X \mathbb{P}$-a.s. To this end, let $\left(X_{n}\right)_{n \in \mathbb{N}}$ be such a sequence and let $X \in L^{\infty}$ be its $\mathbb{P}$-a.s. limit. Then, $Y_{n}:=$ $\operatorname{ess~sup~}_{m \geq n}\left|X_{m}-X\right|$ is bounded and decreases to 0 . By bounded continuity, for every $\delta>0$ there is an $n(\delta) \in \mathbb{N}$ such that

$$
\psi\left(\left|X_{n}-X\right| / \delta\right) \leq \psi\left(Y_{n} / \delta\right) \leq 1 \quad \text { for } n \geq n(\delta) .
$$

Hence, if $n \geq n(\delta)$, then $\left\|X_{n}-X\right\|_{\psi} \leq \delta$.

Step 2. According to Proposition 3.2 (iii), each element $\mu \in L^{\psi *}$ if restricted to $L^{\infty}$ may be identified with a finitely additive signed measure (which we also denote by) $\mu: \mathcal{F} \rightarrow \mathbb{R}$ such that $\mu(A)=0$ whenever $\mathbb{P}(A)=0$ (see [10, Theorem A.50). We claim that $\mu$ must be $\sigma$-additive, or in other words that $\mu\left(A_{i}\right) \rightarrow 0$ for any sequence of sets $\left(A_{i}\right)_{i \in \mathbb{N}} \subset \mathcal{F}$ such that $A_{i} \downarrow \emptyset$ (i.e. $A_{i} \supset A_{i+1}, \mathbb{P}\left(A_{i}\right)>0$ for all $i \in \mathbb{N}$ and $\left.\bigcap_{i \in \mathbb{N}} A_{i}=\emptyset\right)$. In order to verify this claim, consider $\mu \in L^{\psi *} \backslash\{0\}$ and a sequence $\left(A_{i}\right)_{i \in \mathbb{N}} \subset \mathcal{F}$ with $A_{i} \downarrow \emptyset$. Recall the following property of normed spaces (see e.g. [1], Lemma 6.14):

$$
\|X\|_{\psi}=\sup _{\|\nu\|_{\psi *}=1}|\langle\nu, X\rangle|,
$$


where $\|\cdot\|_{\psi *}$ denotes the operator norm on $L^{\psi *}$. In conjunction with Step 1 of this proof we have

$$
\frac{\left|\left\langle\mu, 1_{A_{i}}\right\rangle\right|}{\|\mu\|_{\psi *}} \leq\left\|1_{A_{i}}\right\|_{\psi} \rightarrow 0 \quad \text { for } i \rightarrow \infty
$$

Hence, we have shown that $\mu$ must be $\sigma$-additive, and thus there is $Z_{\mu} \in L^{1}$ such that $\langle\mu, X\rangle=E\left[Z_{\mu} X\right]$ for all $X \in L^{\infty}$. Moreover, due to Proposition 3.2[(iv), for all $X \in L_{+}^{\psi}$ we have that

$$
E\left[Z_{\mu}^{+} X\right]=\lim _{n \rightarrow \infty} E\left[Z_{\mu}^{+}(X \wedge n)\right]=\lim _{n \rightarrow \infty}\left\langle\mu,(X \wedge n) 1_{\left\{Z_{\mu} \geq 0\right\}}\right\rangle \leq\|\mu\|_{\psi *}\|X\|_{\psi} .
$$

Hence, $Z_{\mu}^{+} \in L^{\psi *}$. Similar arguments yield $Z_{\mu}^{-} \in L^{\psi *}$, and thus $Z_{\mu} \in L^{\psi *}$. Uniqueness of $Z_{\mu}$ is clear.

\section{Proof of Theorem 2.9}

Step 1. Due to monotone convergence, Remark 2.2 and ordinary duality, for all $X \in L^{0}$ and all $Z \in \operatorname{dom} \psi^{*} \subset L_{+}^{1}$ such that $E[Z X]>-\infty$, we have

$$
E[Z X]-\psi^{*}(Z)=\lim _{n \rightarrow \infty} \lim _{m \rightarrow \infty} E[Z((-m) \vee X \wedge n)]-\psi^{*}(Z) \leq \psi(X) .
$$

Therefore, and by Theorem 2.1 .

$$
\psi(X)=\sup _{Z \in L_{+}^{1}}\left(E[Z X]-\psi^{*}(Z)\right), \quad X \in L^{0} .
$$

Step 2. Note that the norms $\|\cdot\|_{C, \psi}=\inf \{\lambda>0 \mid \psi(|X| / \lambda) \leq C\}, C>0$, on $L^{\psi}$ are all equivalent and $\|\cdot\|_{1, \psi}=\|\cdot\|_{\psi}$. Moreover, note that $X \in \mathcal{B}_{C, \psi}(1):=\left\{Y \in L^{\psi} \mid\right.$ $\left.\|Y\|_{C, \psi}<1\right\}$ for some $C>0$ is equivalent to the existence of some $\epsilon>0$ such that $\psi((1+\epsilon)|X|) \leq C$, which by convexity implies $(1+\epsilon) \psi(|X|) \leq \psi((1+\epsilon)|X|) \leq C$. Consequently, $\left.\mathcal{B}_{C, \psi}(1) \subset \operatorname{int} \operatorname{dom} \psi\right|_{L \psi}$ for all $C>0$. Taking the union over all $C$ shows that every $X \in L^{\psi}$ for which there is an $\epsilon>0$ such that $\psi((1+\epsilon)|X|)<\infty$ lies in the interior of $\left.\operatorname{dom} \psi\right|_{L^{\psi}}$.

Step 3. Let $X \in \operatorname{dom} \psi$ such that $X^{+} \in L^{\psi}$. Define $\psi_{-X^{-}}(Y):=\psi\left(Y-X^{-}\right)$, $Y \in L^{\psi}$. Note that $\psi_{-X^{-}}$is a monotone l.s.c. convex function on $L^{\psi}$ such that $X^{+} \in$ dom $\psi_{-X^{-}}$. Thus, according to the Fenchel-Moreau theorem (Proposition 3.1 in [8]) and monotonicity we have that

$$
\psi_{-X^{-}}\left(X^{+}\right)=\sup _{\mu \in L_{+}^{\psi *}}\left(\left\langle\mu, X^{+}\right\rangle-\psi_{-X^{-}}^{*}(\mu)\right) .
$$

For $\mu \in L_{+}^{\psi *}$, let $Z_{\mu}$ be as in Proposition 3.4. Since by monotone continuity

$$
\begin{aligned}
\sup _{Y \in L^{\infty}}\left(E\left[Z_{\mu} Y\right]-\psi\left(Y-X^{-}\right)\right) & =\sup _{Y \in L^{\infty}}\left(\lim _{n \rightarrow \infty} E\left[Z_{\mu} Y\right]-\psi\left(Y-\left(X^{-} \wedge n\right)\right)\right) \\
& \leq \liminf _{n \rightarrow \infty} \sup _{Y \in L^{\infty}}\left(E\left[Z_{\mu} Y\right]-\psi\left(Y-\left(X^{-} \wedge n\right)\right)\right) \\
& \leq \sup _{Y \in L^{\infty}}\left(E\left[Z_{\mu} Y\right]-\psi\left(Y-X^{-}\right)\right)
\end{aligned}
$$


all the inequalities must be equalities. Hence, noting that $\mu \in L_{+}^{\psi *}$ implies $Z_{\mu} \geq 0$, we may do the following computation:

$$
\begin{aligned}
\psi_{-X^{-}}^{*}(\mu) & \geq \sup _{Y \in L^{\infty}}\left(E\left[Z_{\mu} Y\right]-\psi\left(Y-X^{-}\right)\right) \\
& =\liminf _{n \rightarrow \infty} \sup _{Y \in L^{\infty}}\left(E\left[Z_{\mu} Y\right]-\psi\left(Y-\left(X^{-} \wedge n\right)\right)\right) \\
& =\liminf _{n \rightarrow \infty} \sup _{Y \in L^{\infty}}\left(E\left[Z_{\mu}\left(Y+\left(X^{-} \wedge n\right)\right)\right]-\psi(Y)\right) \\
& =\psi^{*}\left(Z_{\mu}\right)+E\left[Z_{\mu} X^{-}\right] .
\end{aligned}
$$

We conclude that if $\psi_{-X^{-}}^{*}(\mu)<\infty$, then $\psi^{*}\left(Z_{\mu}\right)<\infty$ and $E\left[Z_{\mu} X^{-}\right]<\infty$.

Step 4. Let $X \in L^{0}$ be such that there is an $\epsilon>0$ with

$$
\psi\left((1+\epsilon) X^{+}\right)<\infty \text { and } \lim _{n \rightarrow \infty} \psi\left(X+\epsilon X 1_{\{X \geq n\}}\right)=\psi(X) .
$$

Then, according to Step 2, $\left.X^{+} \in \operatorname{int} \operatorname{dom} \psi\right|_{L^{\psi}}$. By monotonicity we infer that $\left.\operatorname{int} \operatorname{dom} \psi\right|_{L^{\psi}} \subset \operatorname{int} \operatorname{dom} \psi_{-X^{-}}$. Since $\psi_{-X^{-}}$is a convex l.s.c. function on a Banach space, it is subdifferentiable over the interior of its domain ( 8 , Lemma 2.5 and Proposition 5.2) and thus in particular subdifferentiable at $X^{+}$. Let $\mu \in L^{\psi *}$ be the subgradient of $\psi_{-X}$ - at $X^{+}$, i.e.

$$
\psi(X)=\psi_{-X^{-}}\left(X^{+}\right)=\left\langle\mu, X^{+}\right\rangle-\psi_{-X^{-}}^{*}(\mu) .
$$

Then,

$$
\begin{aligned}
\psi\left(X+\epsilon X^{+} 1_{\{X \geq n\}}\right) & =\psi_{-X^{-}}\left(X^{+}+\epsilon X^{+} 1_{\{X \geq n\}}\right) \\
& \geq \epsilon\left\langle\mu, X^{+} 1_{\{X \geq n\}}\right\rangle+\left\langle\mu, X^{+}\right\rangle-\psi_{-X^{-}}^{*}(\mu) \\
& \geq \epsilon\left\langle\mu, X^{+} 1_{\{X \geq n\}}\right\rangle+\psi(X) .
\end{aligned}
$$

Hence, taking limits for $n \rightarrow \infty$ and by (4.1) we obtain that

$$
\left\langle\mu-Z_{\mu}, X^{+}\right\rangle=\lim _{n \rightarrow \infty}\left\langle\mu, X^{+} 1_{\{X \geq n\}}\right\rangle \leq 0 .
$$

In conjunction with Steps 1 and 3 we have

$$
\psi(X)=\left\langle\mu, X^{+}\right\rangle-\psi_{-X^{-}}^{*}(\mu) \leq E\left[Z_{\mu} X\right]-\psi^{*}\left(Z_{\mu}\right) \leq \psi(X) .
$$

\section{REFERENCES}

[1] Aliprantis, C.D. and Border, K.C. (1999), Infinite Dimensional Analysis, Springer. MR.1717083 (2000k:46001)

[2] Brannath, W. and Schachermayer, W. (1999), A Bipolar Theorem for Subsets of $L^{0}(\Omega, \mathcal{F}, \mathbb{P})$, Séminaire de Probabilités XXXIII, Springer Lecture Notes in Mathematics, Vol. 1709, 349354. MR.1768009 (2001d:46019)

[3] Cheridito, P., Delbaen, F., Kupper, M. (2005), Coherent and convex risk measures for bounded cadlag processes, Finance and Stochastics, 9(3). MR2211713 (2006j:91176)

[4] Cherny, A. and Kupper, M. (2008), Divergence Utilities, preprint.

[5] Delbaen, F. (2002), Coherent risk measures on general probability spaces. Essays in Honour of Dieter Sondermann, Springer-Verlag. MR 1929369

[6] Drapeau, S. and Kupper, M. (2010), Risk Preferences and Their Robust Representation, preprint.

[7] Dunford, N. and Schwartz, J. (1976), Linear Operators. Part I: General theory, Interscience Publishers. MR1009162 (90g:47001a)

[8] Ekeland, I. and Témam, R. (1999), Convex Analysis and Variational Problems, Chapter I, SIAM. MR1727362 (2000j:49001)

[9] Filipović, D., Kupper, M., Vogelpoth, N. (2009), Separation and Duality in Locally $L^{0}$ Convex Modules, Journal of Functional Analysis, 256, 3996-4029. MR2521918 
[10] Föllmer, H. and Schied, A. (2004), Stochastic Finance, An Introduction in Discrete Time, 2nd Edition, de Gruyter Studies in Mathematics, 27. MR2169807(2006d:91002)

[11] Guo, T. (2010), Relations between some basic results derived from two kinds of topologies for random locally convex modules, Journal of Functional Analysis, 258, 3024-3047. MR 2595733

[12] Guo, T. (2010), Recent progress in random metric theory and its applications to conditional risk measures, arXiv:1006.0697v7.

[13] Halmos, P.R. and Savage, L.J. (1949), Application of the Radon-Nikodym Theorem to the Theory of Sufficient Statistics. Annals of Mathematical Statistics, 20, 225-241. MR0030730 $(11: 42 \mathrm{~g})$

[14] Kardaras, C. and Žitković, G. (2010), Forward-convex convergence of sequences in $L_{+}^{0}$, preprint.

[15] Kardaras, C. (2010), Strictly positive support points of convex sets in $L_{+}^{0}$, preprint.

[16] Krein, M. and Šmulian, V. (1940), On regulary convex sets in the space conjugate to a Banach space. Ann. Math. (2), 41, 556-583. MR0002009 (1:335e)

[17] Svindland, G. (2010), Subgradients of Law-Invariant Convex Risk Measures on $L^{1}$, Stat. Decisions, 27, 169-199. MR2662721

Mathematics Institute, Humboldt University Berlin, Unter den Linden 6, D-10099 Berlin, Germany

E-mail address: kupper@math.hu-berlin.de

École Polytechnique Fédérale de Lausanne, CDM SFi CSF - EXTRA 218, CH-1015

Lausanne, Switzerland

E-mail address: gregor.svindland@epfl.ch

Current address: Department of Mathematics, University of Munich, Theresienstr. 39, D-80333 Munich, Germany

E-mail address: svindla@math.lmu.de 